\title{
Development of Techniques Enabling Suborbital Small Object Capture Around Low Gravity Asteroids
}

\author{
Leonard D. Vance* and Jekan Thangavelautham ${ }^{\dagger}$
}

\begin{abstract}
The exploration of small body asteroids provides direct access to the primitive building blocks of our solar system. Sample and return missions enable chemical and radioisotope studies which not only provide evidence for the formation of the solar system, but also a basic understanding of where resources might be found for future exploitation. The touch-down and sample techniques established by Hayabusa-2 and OSIRIS-REx accomplish this mission by physically touching down on the asteroid and collecting samples into a basket extended via a probe from the bottom of the spacecraft. This technique has been demonstrated to work, but contains a high cost in both mission operations as well as the size and complexity of the collection mechanism itself. This paper explores an alternative sample and return technique by exploiting the recent discovery of regolith particle ejections from Bennu. Particles ejected from the surface of Bennu are typically $1 \mathrm{~cm}$ in size and spend several hours in flight, suggesting the possibility that nanospacecraft deployed from the mother-spacecraft could chase down, collect and return with the sample with minimal sensor and delta-V capability. Key aspects of this mission are developed to reduce risk, and an overall mission concept is developed to establish plausibility.
\end{abstract}

\section{Introduction}

Asteroid sample and return missions provide scientists with direct access to materials formed during contraction of our original solar planetary disc. These missions will also play an important part in resource prospecting, especially for water which can be used a propellant for interplanetary spacecraft [65]. Chemical and isotopic analysis of these samples provide the gold standard for understanding the age, history and evolution of the overall solar system. Several missions have therefore attempted this, including Hayabusa 1 and 2, OSIRIS-REx, and NASA's Stardust, but the challenges involved in gathering surface samples has proven more difficult than perhaps originally expected. The combination of very low gravity, relatively high spin rates, and the unpredictability of the surface composition have proven difficult to predict, and the results from initial missions have been mixed. Improvements in retrieval techniques could plausibly reduce mission risks and costs significantly. Mother-Daughter architectures have also been explored, exploring the plausibility of daughter vehicles to undertake local missions in swarms which would would not be plausible with a single spacecraft [64]. This also avoid a single daughter craft having to have long-range communications such as using inflatable antennas [66].

In December 2018, OSIRIS-Rex arrived at the near-earth asteroid Bennu as a prelude to touching down and retrieving such a sample. The initial survey of the asteroid provided two significant surprises. The first involved the lack of fine particulate matter on the surface, and the second was the discovery that Bennu is a "live" asteroid, with low velocity objects erupting regularly from the surface. The first discovery provides a significant challenge to the mission as the sample system is designed to collect relatively small particles, while the second suggests that future sample and returns could use small deployed spacecraft, capturing the expelled samples mid-flight before returning to the mother spacecraft.

This paper explores the design of such a mission, which is plausibly less complex than the sample and return techniques currently utilized. Techniques developed for such a mission would also enable other high value missions such as low earth orbit debris capture and potential on-orbit servicing by nano-spacecraft.

Several areas of novel research must be explored to develop a plausible system design. To begin with, characteristics of the expelled objects must be established. Positions, velocities, sizes and eruption frequencies are needed. It is credible that the movement of these objects in part explain the "double-top" shape of spinning rubble pile asteroids, so it may be conversely possible that the "double-top" shape implies the existence of object expulsions, and therefore indicates opportunities to collect samples without touching the surface.

*PhD Candidate, Aerospace and Mechanical Engineering, University of Arizona, 1130 N Mountain Ave, Tucson, AZ 85721

${ }^{\dagger}$ Assistant Professor, Aerospace and Mechanical Engineering, University of Arizona, 1130 N Mountain Ave, Tucson, AZ 85721 


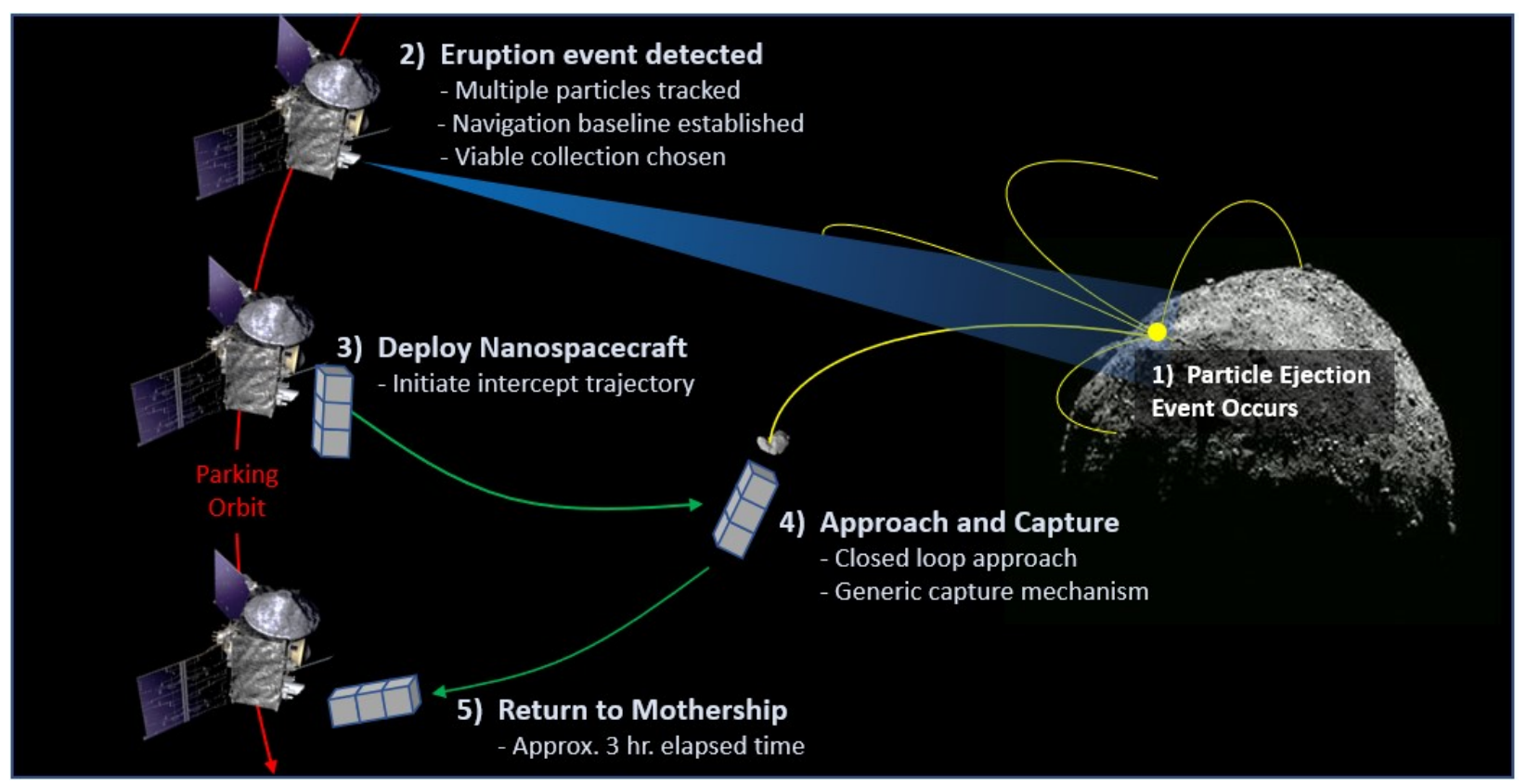

Fig. 1 Aspects of the proposed mission. Nano-spacecraft are deployed from a parking orbit to collect ejected particles and return to the mother spacecraft

The design of a temporarily deployed nano-spacecraft to capture expelled objects provides significant technical challenges. Each vehicle must be able to navigate, guide, collect, return and dock with the parent spacecraft within the timeframe of a typical particle trajectory. To begin with, autonomous navigation can be established using passive line of sight histories to the targeted object. The inertial line of sight history between two orbital objects provides sufficient information to establish estimate ephemeris for both objects, and this can be realized with a modified Extended Kalman Filter (EKF) formulation, possibly simplified to a constant gain model to reduce processor utilization. The resulting implementation of closed loop guidance using the aforementioned navigation output with Lambert's method is well known but computationally expensive, and is thus replaced with research towards a perceptron based network trained for operation within any particular asteroid's gravitational field.

Spacecraft control involves both attitude and position control, and would be exercised via a six degree of freedom thruster system possibly including reaction wheels for fine control. The concept of a minimal redundant thruster configuration for nanospacecraft is explored via null vector analysis, leading to candidate minimal thruster systems with single failure redundancy. Finally, a generic grappling system is discussed for a non-cooperative target, with possible application to wider missions such as debris removal from low earth orbit. The overall proposed mission is summarized in Figure 1

\section{Ejected particle characterization on Bennu}

The process of rubble pile asteroid formation was discussed by Michel [13] in 2001, and Guibot and Scheeres [11] in 2003, but innovations in deep space radar imaging led to the confirmation in 2006 [17], [23] that asteroid (66391) 1999 KW4 has a top-shape with a nearly constant slope of about 35 degrees from mid latitudes down to the equator. It is a fast-rotator, with a period $2.8 \mathrm{hr}$ and a small moon just off the equator suggesting recent mass shedding [23]. Subsequent radar observations of (341843) 2008 EV5 [18], (29075) 1950 DA [16] and (10195) Bennu [24] show that top-shapes are common for near-earth asteroids. Arrival of OSIRIS-REx and Hayabusa-2 confirmed this [31] for both (10195) Bennu and (162173) Ryugu [9] respectively, providing detailed 3D models to enable detailed analysis

Centripetally-induced landslides caused by solar-pressure induced YORP spin-up have been proposed to explain current observations [11], [8]. As more data have become available, these ideas have been developed analytically [2], [3], [6], [10], [22] and avalanche failure mechanisms have been explored [29], [27]. Particle models [19], [26], [30], some including cohesion [14], [15], have been applied to the problem. There have also been attempts to simulate low speed collisions as a possible origin for top-shapes. Simulations of impacts between two near 


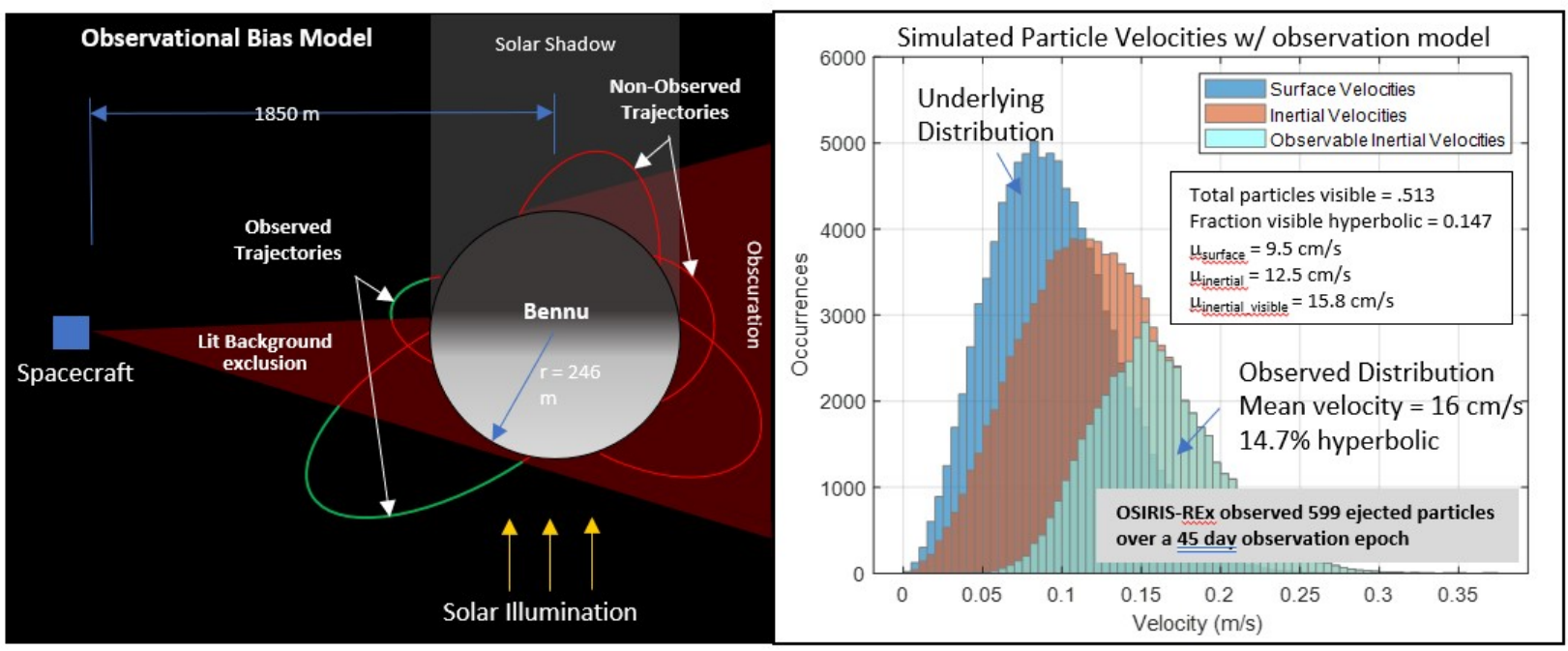

Fig. 2 Particle frequency and velocity distributions derived from OSIRIS-REx observations provide a basis for the proposed mission

equal mass rubble piles, where friction and porosity is included [5], can produce final populations where top-shapes are common. Additionally, the direct measurements of the YORP induced angular acceleration on Bennu [1], [20], and its sensitivity to variations in configuration [21] provide an understanding of the possible chaotic nature of Bennu's accelerating spin rate.

The discovery in 2019 by NASA's OSIRIS-REx mission that the 500-metre diameter carbonaceous rubble pile Bennu is an active asteroid, regularly ejecting objects from the surface [4], suggests the possibility that objects can be captured in flight without approaching the asteroid surface. Some 599 objects were observed by OSIRIS-REx over a 45 day observation period following its initial approach to the asteroid, providing a mean of 13 objects observed per day. When a simulation experiment is executed accounting for the obscuration of most of the actual particles leaving the surface, the actual launch frequency is about 26 particles per day with a mean launch velocity of $9.5 \mathrm{~cm} / \mathrm{s}$ as shown in Figure 2. The resulting flight time for an average particle is about 1.5 hours given Bennu's very low gravity. This ensures that particles will be available for sampling on a near daily basis.

\section{Own-Ship and Target Navigation with a Single Passive Line-Of-Sight Sensor}

Navigation techniques using passive line-of-sight measurements are well known within the guidance, navigation and control community. The ability to infer ephemeris information of an orbital object by tracking it from a known position is well understood, as is the ability to infer one's own ephemeris by tracking an object with known position and velocity. Here we show that it is possible to infer both own-ship and tracked-object ephemeris given knowledge of the gravitational influences both are subjected to.

Figure 3 shows the general nature of the problem. It is well established that position and velocity data for an overhead satellite can be extracted from passive line of sight measurements from the ground. Observability of range is inferred from the gravitational attraction of the orbiting object. Likewise, as a simple extension, it is also possible to do the same job from an orbiting satellite. If you know your own position and velocity, you can infer the position and velocity of another satellite given a time history line of sight measurements.

With some additional thought, it is possible that the position and velocity of both the tar-get and observer satellites can be inferred from a single line of sight history. With some exceptions, it can be asserted that any line of sight history between two objects gravitation-ally influenced by a common source is the result of only one specific target and home satellite trajectory.

\section{A. Method}

The Extended Kalman Filter (EKF) is a well-known, pseudo-optimal predictor-corrector filter widely used for a wide variety of aerospace purposes. This section shows the adaptation of the basic filter structure for this particular 
Method 1: Position and Velocity

inferred from line of sight

measurements from ground

station with known position

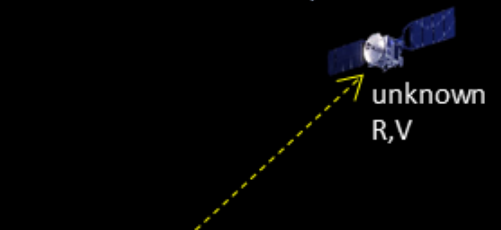

Known

$\mathrm{R}, \mathrm{V}$
Method 2: Position and Velocity inferred from line of sight measurements from satellite with known position and velocity
Method 3: (Subject of this Section) Position and Velocity of both satellites inferred from line of sight history

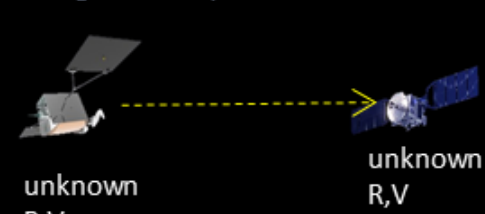

$\mathrm{R}, \mathrm{V}$ unknown
Known

$\mathrm{R}, \mathrm{V}$

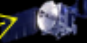

unknown

$\mathrm{R}, \mathrm{V}$

\section{R,} .

.
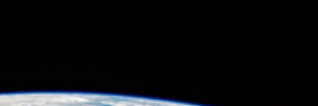

(n)

Fig. 3 The line of sight history between two objects under the influence of a common gravitational body is sufficient to estimate the position and velocity of both

problem. The established form of the EKF [32] starts by propagating the current estimate and covariance matrices forward by one timestep.

$$
\begin{gathered}
\hat{\boldsymbol{x}}(k+1 \mid k)=\hat{\boldsymbol{x}}(k \mid k)+\int_{t_{k}}^{t_{k+1}} f\left[\hat{x}\left(t \mid t_{k}\right), \boldsymbol{u}^{*}(t), t\right] d t \\
P(k+1 \mid k)=\Phi(k+1 \mid k) P(k \mid k) \Phi^{\prime}(k+1, k)+Q_{d}(k)
\end{gathered}
$$

This followed by calculation of the Kalman gains:

$$
\boldsymbol{K}(k+1)=\boldsymbol{P}(k+1 \mid k)\left[\boldsymbol{H}_{x}^{\prime}(k+1) * \boldsymbol{P}(k+1 \mid k) \boldsymbol{H}_{x}^{\prime}(k+1)+\boldsymbol{R}(k+1)\right]^{-1}
$$

The state vector estimate can then be updated:

$$
\hat{x}(k+1 \mid k+1)=\hat{x}(k+1 \mid k)+\boldsymbol{K}(k+1)\left\{\boldsymbol{z}(k+1)-\boldsymbol{h}\left[\hat{\boldsymbol{x}}(k+1 \mid k), \boldsymbol{u}^{*}(k+1), k+1\right]-\boldsymbol{H}_{u}(k+1) \delta \boldsymbol{u}(k+1)\right\}
$$

Finally, also update the covariance matrix:

$$
\boldsymbol{P}(k+1 \mid k+1)=\left[\boldsymbol{I}-\boldsymbol{K}(k+1) \boldsymbol{H}_{x}(k+1)\right] \boldsymbol{P}(k+1 \mid k)
$$

The definition of these parameters are consistent with normal EKF usage:

$$
\begin{aligned}
& \quad \hat{\boldsymbol{x}}=\text { state estimate } \\
& k=\text { current time step } \\
& k+1=\text { next time step } \\
& \boldsymbol{f}=\text { function propagating state estimate } \hat{\boldsymbol{x}} \text { in time } \\
& t=\text { time } \\
& \boldsymbol{u}^{*}=\text { nominal input } \\
& \boldsymbol{P}=\text { Covariance matrix } \\
& \boldsymbol{\Phi}=\text { Linearized time propagation matrix } \\
& \boldsymbol{Q}_{\boldsymbol{d}}=\text { State error propagation matrix } \\
& \boldsymbol{K}=\text { Kalman gain matrix } \\
& \boldsymbol{h}=\text { function taking state vector to measurements } \\
& \boldsymbol{H}_{\boldsymbol{x}}=\text { Jacobean of } \boldsymbol{h} \text { w/r to state vector } \hat{\boldsymbol{x}} \\
& \boldsymbol{H}_{\boldsymbol{u}}=\text { Jacobean of } \boldsymbol{h} \text { w/r to external inputs } \\
& \boldsymbol{I}=\text { Identity matrix }
\end{aligned}
$$




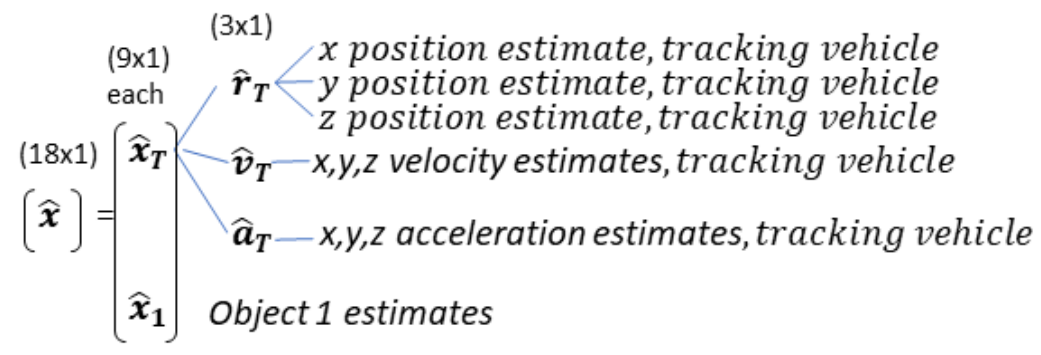

Fig. 4 The line of sight history between two objects under the influence of a common gravitational body is sufficient to estimate the position and velocity of both

The function $\boldsymbol{u}^{*}$ and its derivatives are zero for the purposes of this exercise since there are no external forces acting on the system, and as such, (1) through (5) can be simplified to give

$$
\begin{gathered}
\hat{\boldsymbol{x}}(k+1 \mid k)=\hat{\boldsymbol{x}}(k \mid k)+\int_{t_{k}}^{t_{k+1}} \boldsymbol{f}\left[\hat{x}\left(t \mid t_{k}\right), t\right] d t \\
P(k+1 \mid k)=\Phi(k+1 \mid k) P(k \mid k) \Phi^{\prime}(k+1, k)+Q_{d}(k)
\end{gathered}
$$

for the propagation equations, and

$$
\begin{gathered}
\boldsymbol{K}(k+1)=\boldsymbol{P}(k+1 \mid k)\left[\boldsymbol{H}_{x}^{\prime}(k+1) * \boldsymbol{P}(k+1 \mid k) \boldsymbol{H}_{x}^{\prime}(k+1)+\boldsymbol{R}(k+1)\right]^{-1} \\
\hat{x}(k+1 \mid k+1)=\hat{x}(k+1 \mid k)+\boldsymbol{K}(k+1)\{z(k+1)-\boldsymbol{h}[\hat{\boldsymbol{x}}(k+1 \mid k)\} \\
\boldsymbol{P}(k+1 \mid k+1)=\left[\boldsymbol{I}-\boldsymbol{K}(k+1) \boldsymbol{H}_{x}(k+1)\right] \boldsymbol{P}(k+1 \mid k)
\end{gathered}
$$

for the update equations.

The state estimation vector contains 3 element position, velocity and accelerations for each object. Since we are tracking one object from a home vehicle, there are 18 elements in the state estimation vector comprising:

The function $f$ is a simple orbital propagator, utilizing the basic gravitational law

$$
\boldsymbol{a}=-\frac{G m_{\text {bennu }} \boldsymbol{r}}{|\boldsymbol{r}|^{3}}
$$

where the r's are taken from the position estimates in the state vector. The corresponding matrix $\Phi$ is the Jacobean of this with respect to $\hat{\boldsymbol{x}}$, giving an $18 \times 18$ matrix. The function $\boldsymbol{h}$ takes state variables and constructs the measurements $z$, and is therefore of the form:

$$
z=\boldsymbol{h}(\hat{\boldsymbol{x}})
$$

Since the measurements for this system are the normalized line of sight vectors from the tracking vehicle to the two asteroids, the resulting function $\boldsymbol{h}$ is

$$
\boldsymbol{h}=\left[\begin{array}{c}
\frac{x-x_{t}}{\sqrt{\left(x-x_{t}\right)^{2}+\left(y-y_{t}\right)^{2}+\left(z-z_{t}\right)^{2}}} \\
\frac{x-x_{t}}{\sqrt{\left(x-x_{t}\right)^{2}+\left(y-y_{t}\right)^{2}+\left(z-z_{t}\right)^{2}}} \\
\sqrt{\left(x-x_{t}\right)^{2}+\left(y-x_{t}\right)^{2}+\left(z-z_{t}\right)^{2}}
\end{array}\right]
$$

The Jacobian of this function with respect to each component of the state estimate vector provides the resulting $6 \times$ $18 \boldsymbol{H}_{\boldsymbol{x}}$ matrix.

The $\boldsymbol{R}$ matrix represents the system measurement noise, and this takes the form of angular uncertainties along the line of sight to the object. The measurement vector $z$ is the normalized line of sight vector the target as given by an 


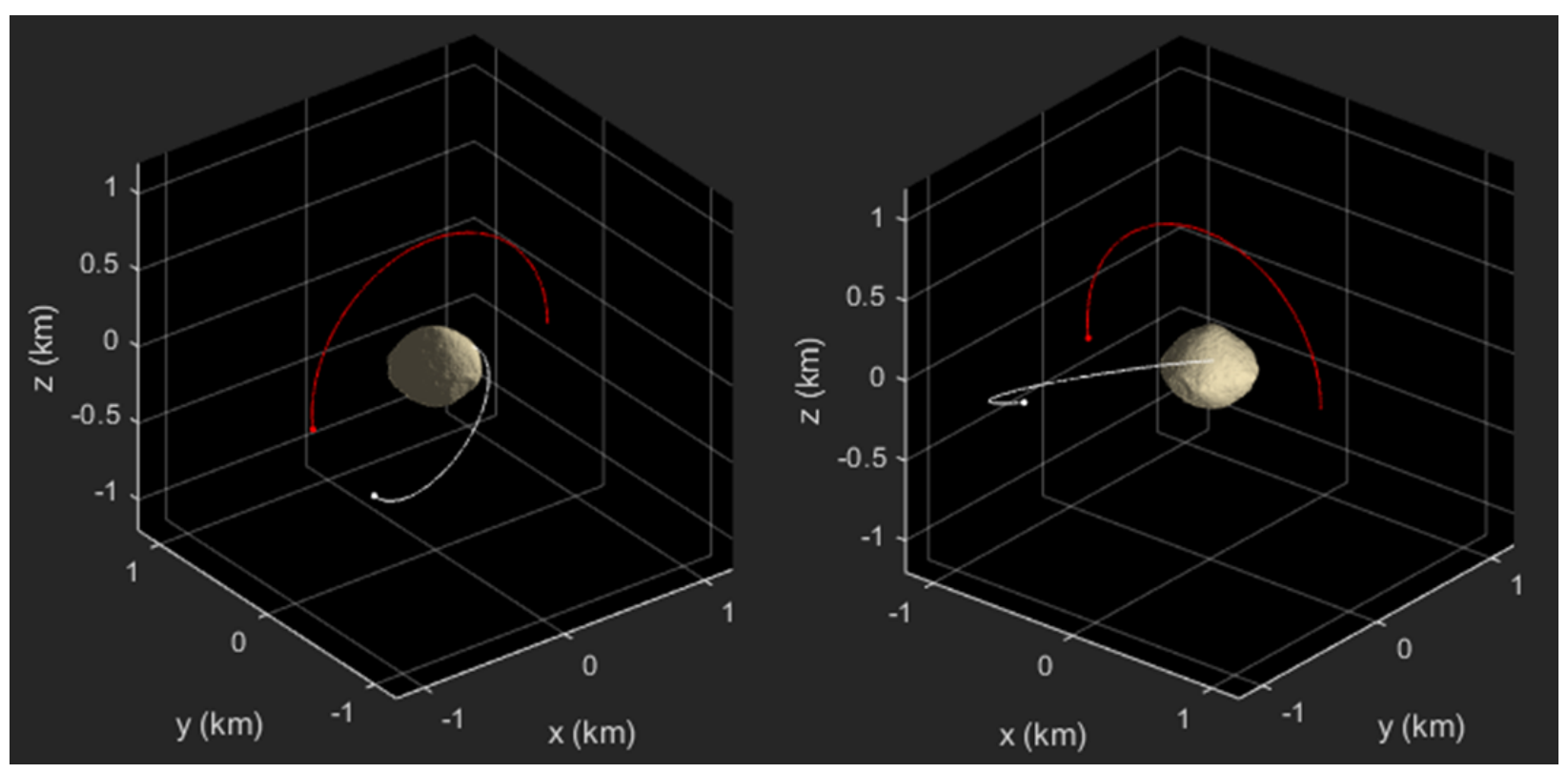

Fig. 5 Proof of concept trajectories for both the spacecraft and the ejected particle around (101955) Bennu

imaging system, so given an angular uncertainty $\epsilon$ in each axis, the covariance matrix for measurement errors to the tracked object, in line of sight coordinates is:

$$
\boldsymbol{R}_{\text {los }}=\left[\begin{array}{ccc}
1 & 0 & 0 \\
0 & \epsilon^{2} & 0 \\
0 & 0 & \epsilon^{2}
\end{array}\right]
$$

This must then be rotated into an inertial coordinate frame used by the rest of the filter. Derivation of the coordinate transform matrix is done by establishing sequential coordinate frame rotation angles about the $\mathrm{z}$ and then $\mathrm{y}$-axes respectively, providing a coordinate transformation matrix of

$$
\boldsymbol{T}_{\text {los }}=\left[\begin{array}{ccc}
\cos \psi \cos \theta & \sin \psi \cos \theta & -\sin \theta \\
-\sin \psi & \cos \psi & 0 \\
\cos \psi \sin \theta & \sin \psi \sin \theta & \cos \theta
\end{array}\right]
$$

Where the sequential angles of rotation about $\mathrm{z}$ and $\mathrm{y}$ axes respectively are:

$$
\begin{gathered}
\psi=\tan ^{-1}\left(\frac{y-y_{T}}{x-x_{T}}\right) \\
\theta=\tan ^{-1}\left(\frac{z-z_{t}}{\sqrt{\left(x-x_{t}\right)^{2}+\left(y-y_{T}\right)^{2}}}\right)
\end{gathered}
$$

This matrix takes a vector from inertial to the line of sight coordinated frame with respect to object 1 . Using a similarity transformation to convert from line of sight to inertial coordinates, we have

$$
\boldsymbol{R}_{\text {inertial }}=\boldsymbol{T}_{\text {los }} \boldsymbol{R}_{\text {los }} \boldsymbol{T}_{\text {los }}^{T}
$$

Finally, the $18 \times 18$ state process noise matrix $Q$ is constructed with arbitrarily small diagonal elements for the purpose of establishing feasibility.

$$
\boldsymbol{Q}=1.0 \times 10^{-30} * \boldsymbol{I}_{18 \times 18}
$$



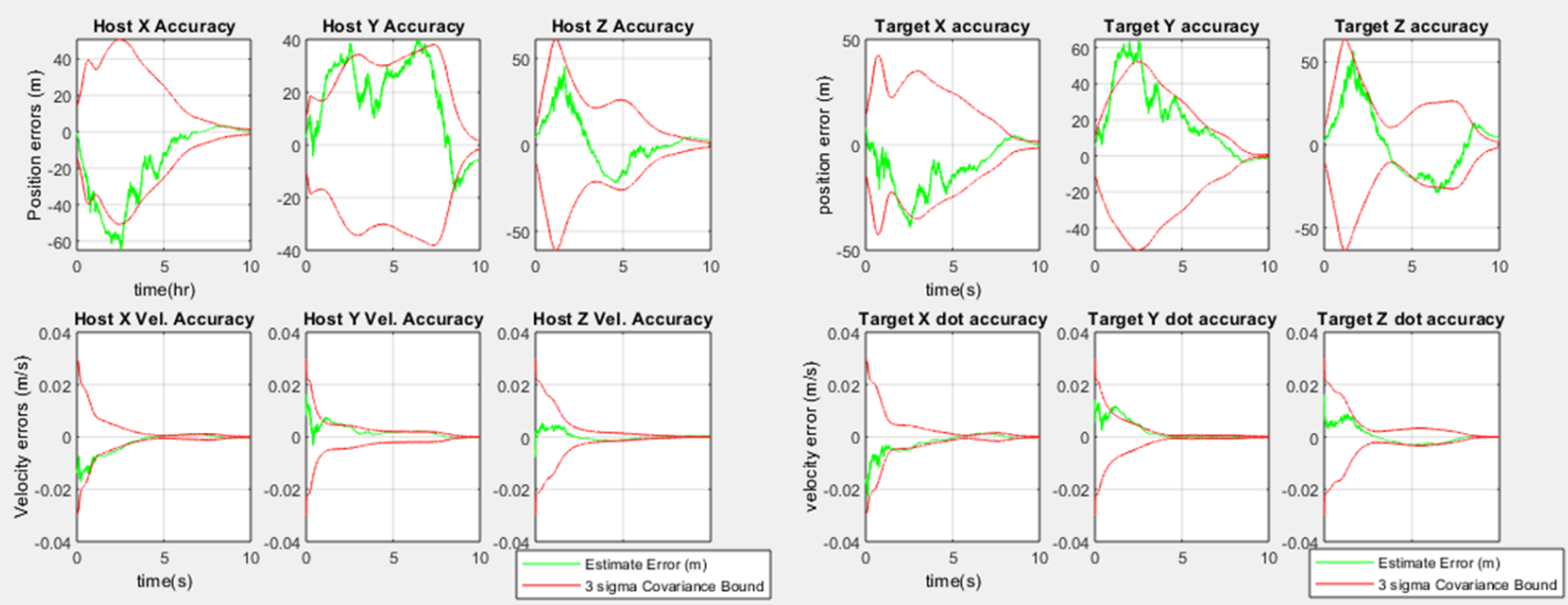

Fig. 6 Convergence of position and velocity estimates for both spacecraft and ejected particle over the 10 hour epoch described by the previous figure

\section{B. Results}

The implementation of the above filter is tested in an environment derived from the physical environment of (101955) Bennu. The specific intent is to show whether autonavigation can be performed from a nanospacecraft that is tasked with capture of an ejected particle from that surface. As proof of concept, the spacecraft is placed in a circular orbit with an $800 \mathrm{~m}$ semi-major axis with 90 degrees inclination. The tracked object ejects from the mid latitudes of Bennu with a velocity of $17.5 \mathrm{~cm} / \mathrm{s}$, and it tracked by the spacecraft for a 10 hour epoch. The trajectories of both objects relative to Bennu is shown in Figure 5

Using only inertial line of sight measurements from the spacecraft to the ejected particle, position and velocity can be established for both simultaneously. Given a reasonable initialization for covariances, the results are shown in Figure 6, illustrating good convergence in position and velocity in all 3 axes, although over a time period of several hours.

\section{Perceptron Based Orbital Guidance in a Low Gravity Asteroid Environment}

\section{A. Introduction}

Lambert's solution is a well studied approach [46], [47] to orbital guidance, providing a near universal iterative solution for transition between two points under the influence of a single gravitational body. The user specifies starting and end points along with transit time and central body gravitational parameter $\mu_{m}$, and the algorithm produces the corresponding velocities at those starting and end locations along with minimum and maximum ranges to the gravitational body. While the algorithm is well known, accurate and almost universal in convergence, it requires a significant number of trigonometric function calls in an iterative framework. In comparison, a feed forward neural network has low computational cost implementation and can theoretically mimic the output of much more complex algorithms if trained correctly.

Neural Network guidance could therefore be used by nanospacecraft to rendezvous and gather the particles emitted by an active asteroid in flight. The discovery in 2019 that Bennu is an active asteroid, and is regularly ejecting particles from its surface [45] makes this concept possible. the frequency, size and velocity of these particle ejections were established to be $1-10 \mathrm{~cm}$ in diameter, 6 to $330 \mathrm{~cm} / \mathrm{s}$ in velocity and were observed to launch episodically with an cumulative frequency of approximately 599 particles over 45 days. The escape velocity from Bennu is about $20 \mathrm{~cm} / \mathrm{s}$, and the time spent by these particles in flight typically is measured in hours before they either escape from gravitational influence, or return to the surface of the asteroid.

In this section, a training set of orbits and their resulting transfer trajectories are established using randomly selected position and velocity parameters around asteroid Bennu as inputs to the Lambert algorithm. Seven separate filters are trained, one each for three axes of starting velocity, ending velocity plus a minimum transition altitude. Figure 7 defines 


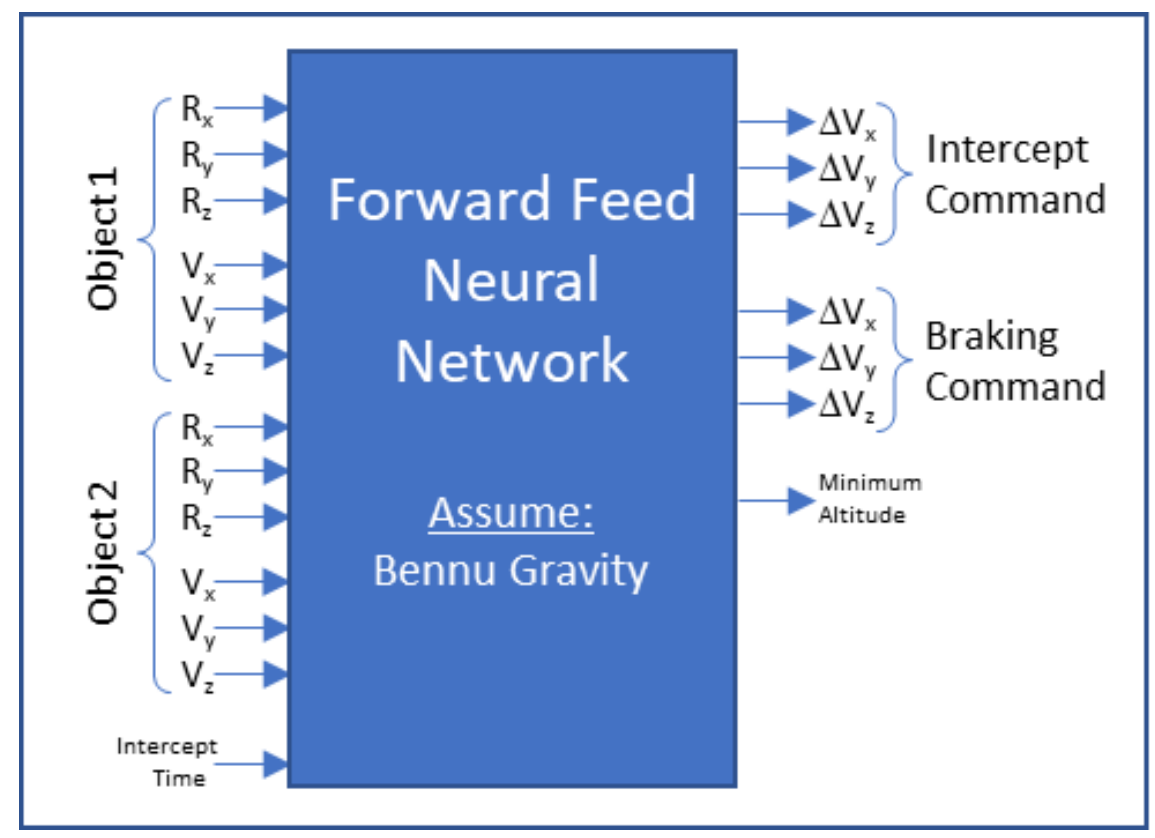

Fig. 7 Definition of the Problem: A neural network approach to lambert guidance is defined to reduce processing requirements for a nanospacecraft attempting to rendezvous an ejected particle around Bennu

the basic outline of the problem, and shows an example of a transfer trajectory derived using Lambert between two objects in orbit around Bennu. A Levenburg-Marquardt algorithm is implemented which shows good convergence with a modest number of hidden nodes and layers. Finally, the resulting trained networks are used to verify performance in an orbital test case.

\section{B. Implementation of Levenburg-Marquardt Optimization}

Research indicates that perhaps the most common training technique for neural networks is the Levenberg-Marquardt algorithm [7]. This method effectively acts as a gradient descent at the beginning of the epoch, and gradually transitions to solve for zero error effectively using a Newton-Raphson style method as the system starts to converge. Using the Jacobean derived for use in gradient descent, the weight update equation can be reformulated as:

$$
w_{i}=\left[P^{T} P+\mu I\right]^{-1} J^{T}\left(y-y_{\text {train }}\right)+W_{i-1}
$$

where $\mu$ is a scaler control variable with value $\mu>0, w_{i}$ is the updated weight vector, $\mathrm{P}$ is the same Jacobean matrix used in the gradient descent method, $\left(y-y_{\text {train }}\right)$ is the error vector and $w_{i-1}$ the previous weight vector. This optimizer approximates a small step gradient descent method when $\mu$ is large, but becomes closer to a Newton-Raphson solver as $\mathrm{mu}$ approaches zero. $\mu$ is adjusted during an optimization run, increasing fractionally if the error function increases, but decreasing fractionally when it is converging. In a nominally converging system, $\mu$ will steadily decrease as the network converges.

This algorithm performs well and converges for all six $\Delta V$ estimates. Figure 8 shows this convergence using this technique for a modest 2 layer system of 16 and 8 hidden nodes respectively. All other values of $\Delta V$ show similar excellent results, though the estimate for minimum altitude during the intercept trajectory has more difficulty converging. Performance starts to fall off significantly with less that 16 nodes in the first hidden layer, but is not visibly improved with increases beyond the nominal values for the two layer system specified as the nominal case. The rms error of this estimator calculates to $0.9 \mathrm{~cm} / \mathrm{s}$, and when implemented as the guidance method, the resulting trajectories can be seen in Figure 9 

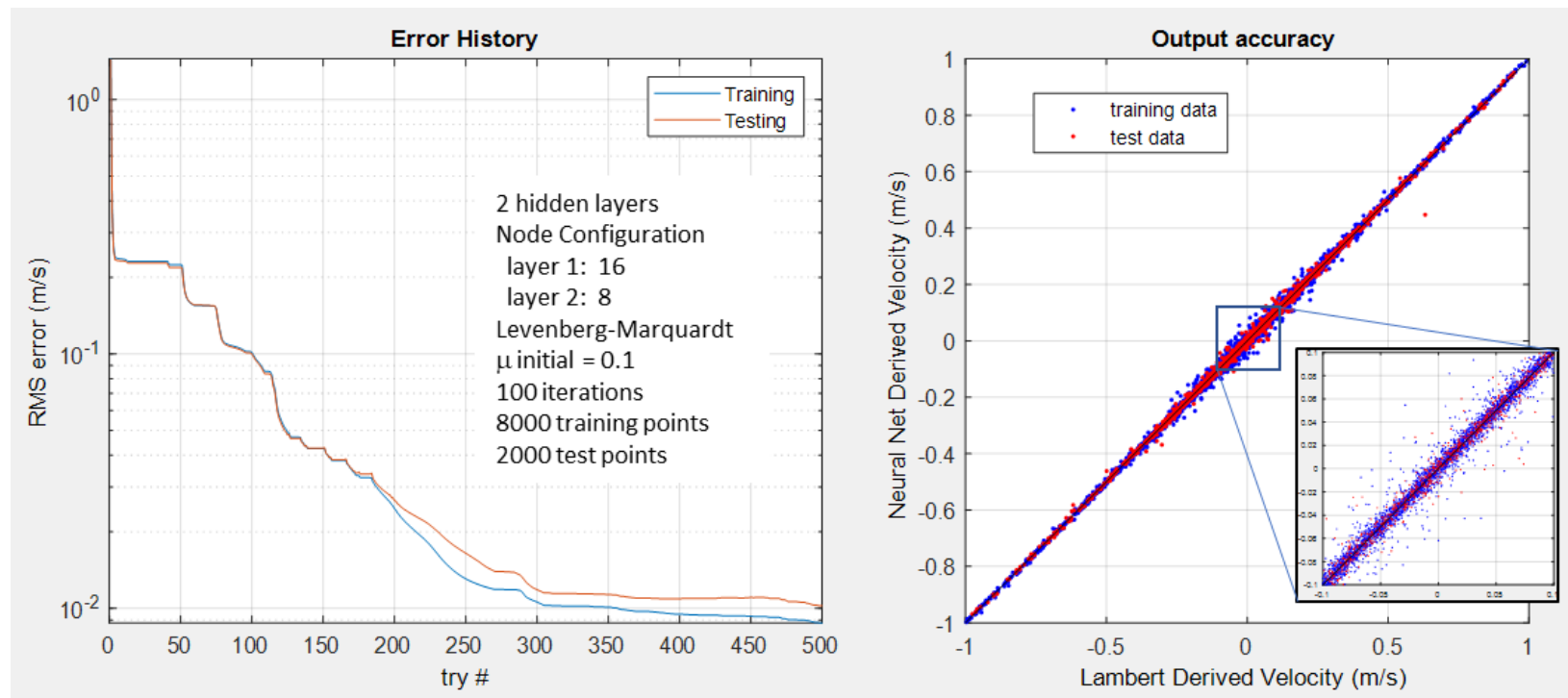

Fig. 8 Levenberg-Marquardt Descent for Lambert's solution converges quickly and accurately for the velocity$\mathbf{X}$ axis, and this behavior is near identical for the other two axes, as well as the velocity required at intercept.

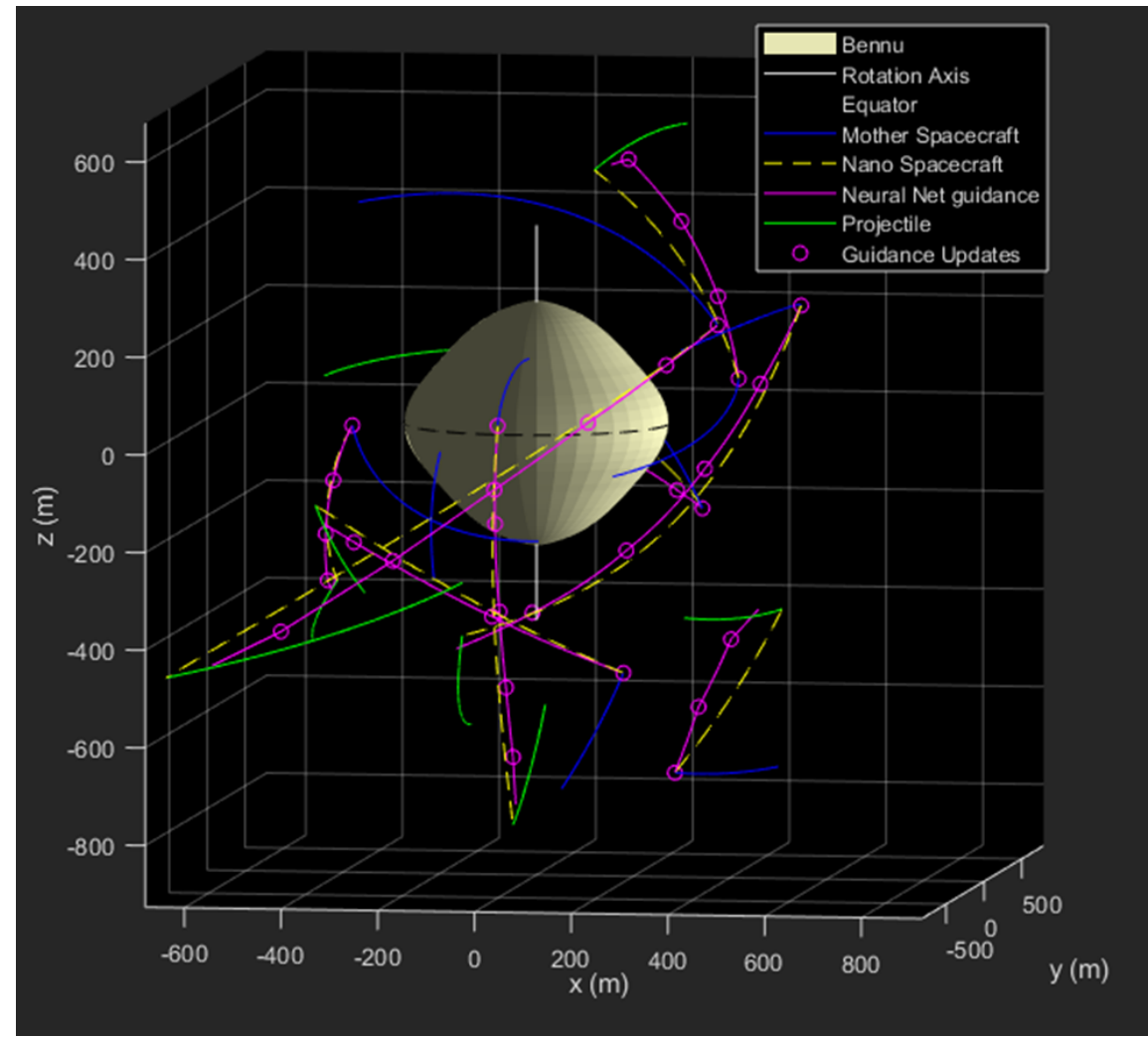

Fig. 9 The overall performance of the resulting neural network guidance is shown, comparing the Lambert derived trajectory (yellow) to the neural network derived trajectory (magenta). Discrete guidance updates using the neural network are calculated and shown at 1250 second intervals (magenta circles) 


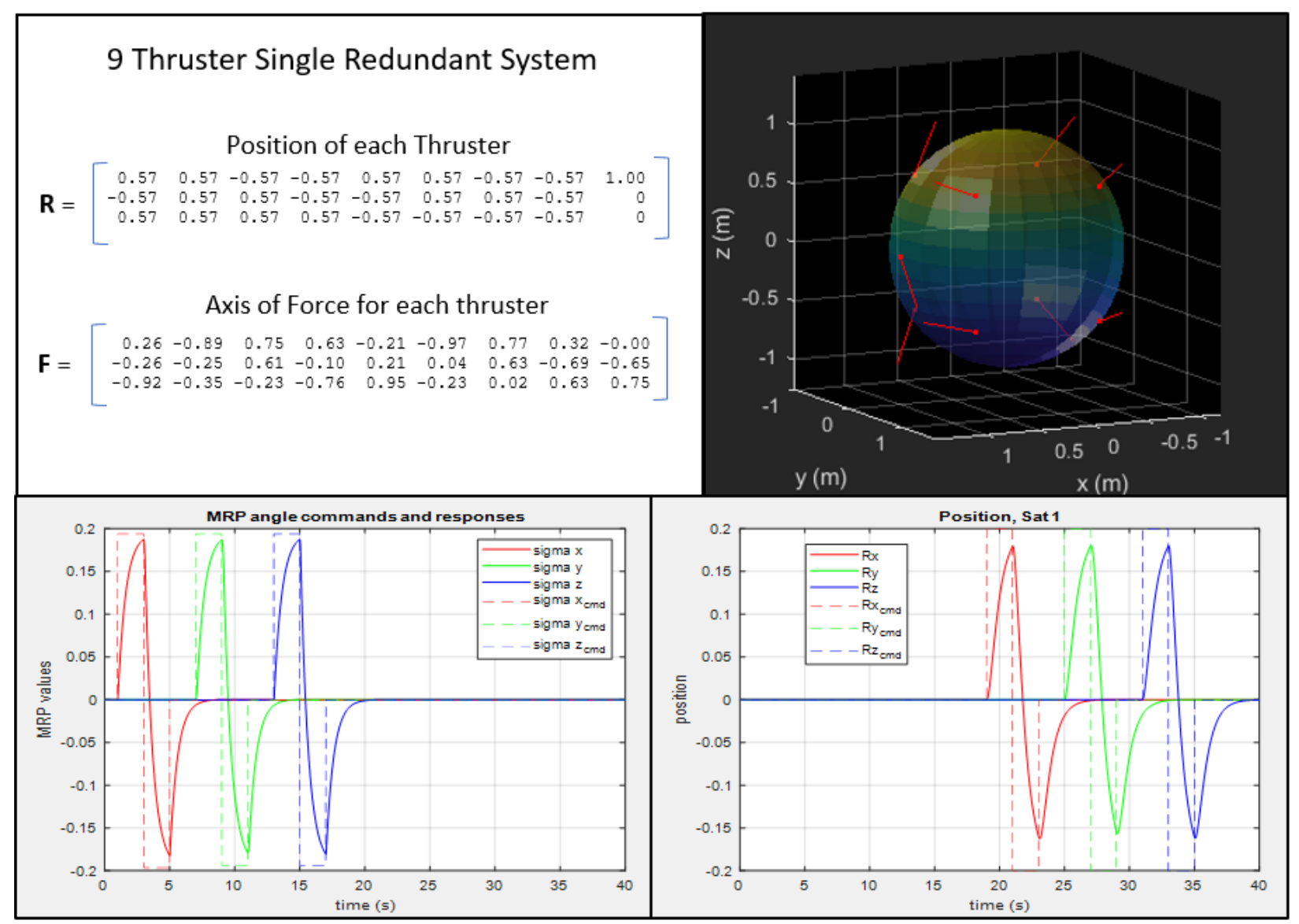

Fig. 10 An example of a fully redundant, six degree of freedom thruster configuration derived via pseudoinverse techniques combined with null vector evaluation. Improvement of placement and orientation via genetic algorithms provides a path towards optimizing nanospacecraft propulsion systems

\section{Thruster Configuration Optimizations}

The size and complexity of a spacecraft is significantly effected by its propulsion configuration. In the case of a nanospacecraft executing the mission of collecting ejected particles from an active asteroid, there would be a requirement for full six degree of freedom motion, and possibly a requirement for redundancy if one of the thrusters fails. The physical volume required for plumbing, valves and expansion nozzles encourages the designer to reduce the individual number of thrusters to the absolute minimum. This section discusses the minimum configuration needed for full 6 axis control of a nanospacecraft, and then the mathematical techniques to show controllability as well as thruster failure redundancy.

This section seeks to establish minimal thruster configurations which maximize effectiveness and while still providing single thruster failure redundancy. The actuation logic for a multiple coupled thruster system can be expressed as:

$$
\left[\begin{array}{l}
\boldsymbol{F}_{\boldsymbol{b}} \\
\boldsymbol{L}_{\boldsymbol{b}}
\end{array}\right]=[\boldsymbol{T}]\left[\boldsymbol{F}_{\boldsymbol{t}}\right]
$$

where $\boldsymbol{F}_{\boldsymbol{t}}$ is a vector containing the thrust values from each thruster at a given time, $\boldsymbol{F}_{\boldsymbol{b}}$ is the resulting three dimensional net thrust in the body frame, $\boldsymbol{L}_{\boldsymbol{b}}$ is the body frame torque vector, and $\boldsymbol{T}$ is the matrix relating thrust values to net forces and torques.

In order to get full three dimensional control of a spacecraft in both linear and rotational space, a minimum of seven thrusters is required, with more needed if redundancy is required. The classical approach to establishing the thruster control levels needed uses the pseudo inverse method such that 


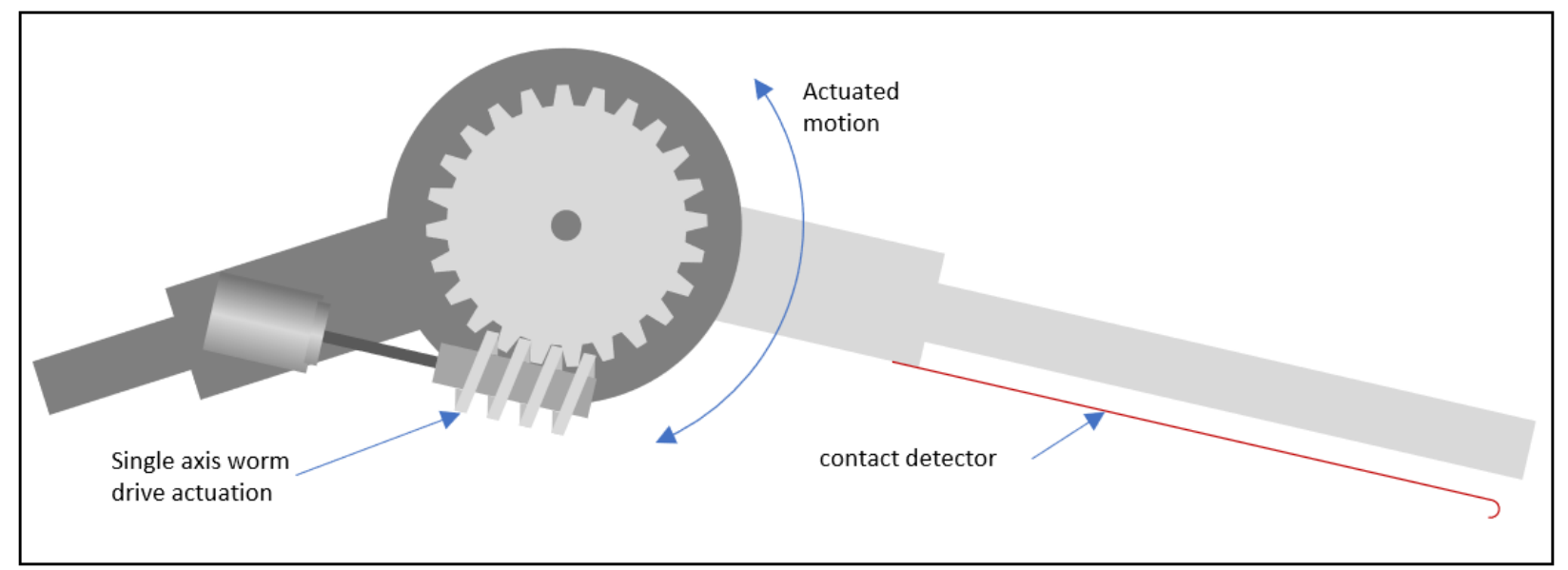

Fig. 11 Link Concept: The candidate link concept uses a miniature worm drive to actuate successive links. A wire is included in the outline of each link which is triggered upon establishing contact with an object.

$$
F_{t}=\left[T^{-1}\right]\left[\begin{array}{l}
F_{b} \\
L_{b}
\end{array}\right]
$$

In order this technique to work, there must also be at least one positive definite null space vector to the $\boldsymbol{T}$ matrix, so that thrust levels derived from the pseudo-inverse method above can be adjusted upwards until all are non-zero. In the case of testing for redundancy, these two conditions must be met for a each subset of the $\boldsymbol{T}$ matrix corresponding to the loss of one thruster.

Preliminary simulations have established minimal configurations of 9 thrusters which provide full 6 degree of freedom control with single failure redundancy, but these thruster configurations are asymmetric. Figure 10 shows an example of such a system configured on a spherical volume which has full six degree of freedom control for both the nominal configuration as well as any given single failures. Time history performance of this configuration is shown in the bottom half of this same figure. The proposed dissertation shall execute the analysis necessary to establish full six degree of freedom control with redundancy with a minimum number of thrusters, including placement and orientations optimized for control effectiveness.

\section{Generic Capture Mechanism}

This concept involves the deployment of multiple grappling arms, each comprised of identical segments with independently controlled single axis rotational joints. each link includes a sensor to establish contact with the object to be grappled. Figure 11 provides an overview of one joint of this proposed grappling system.

Upon establishing position and rate matching along center of rotation of the object to be grappled, the spacecraft starts to extend the adaptive grappler link by link, stopping each time contact is detected with that link, and then stepping to the next link. In this fashion, the target object is slowly enveloped in a low-force, controlled fashion until the entire grappling arm is deployed. This approach is shown in a planar view in Figure 12

The control for this concept needs to be expanded to include the algorithms necessary to establish a rippling motion for the as yet undeployed links so that 1) the amount of energy necessary to deploy the system is reduced, and 2) to reduce the probability that grappler might hit obstructions out beyond the minimal extent of the object being captured. 6-degree of freedom simulation of this concept and the capture sequence will be executed and optimized for system performance leading to a conceptual design.

\section{Conclusions}

In light of the complexity and risks associated with the sampling experience of Hayabusa 1 and 2 as well as OSIRIS-REx, establishing alternative approaches to obtain samples from small asteroids is a beneficial exercise. The discovery of regularly ejected particles from Bennu suggests the possibility that most if not all top shaped near Earth 

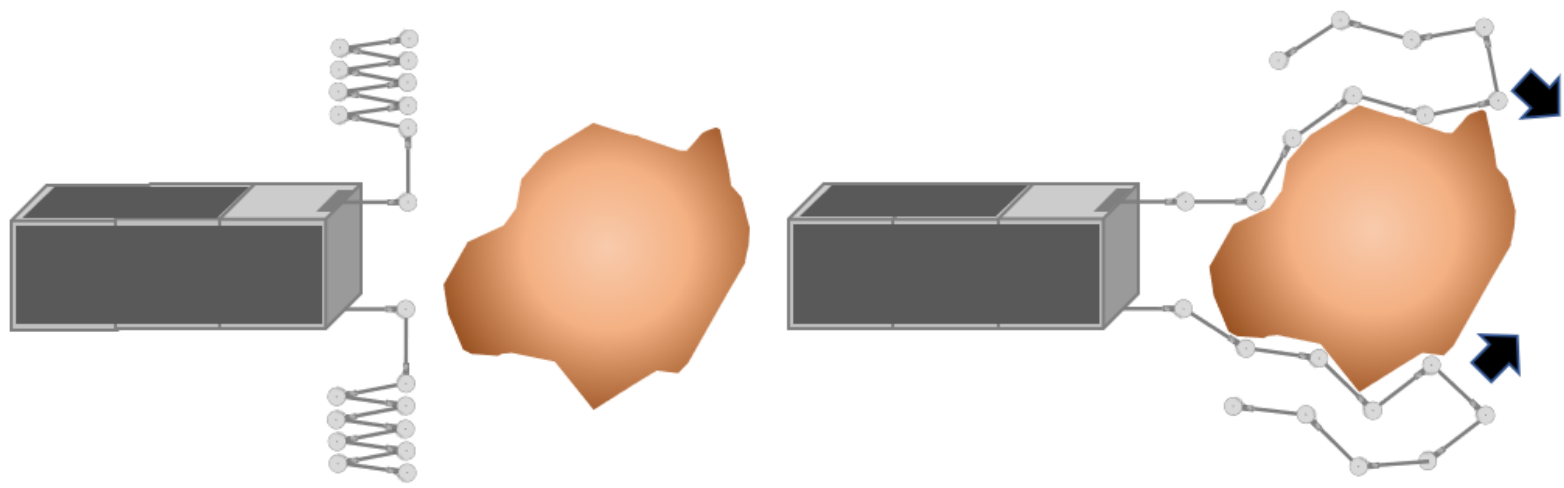

Fig. 12 Concept of Operations: Upon establishing stable proximity with the object to be captured, the spacecraft establishes an approach vector along the spin axis of the object, and deploys the grappler arms one link at a time, where each link stops when sensing contact with the object, and subsequent links then rotate forward, slowly enveloping the object to be captured.

asteroids are active in some similar fashion, enabling the possibility that sampling could be done by capturing these particles in flight and returning them to Earth. This dissertation proposal documents the development of key technologies enabling this mission.

Characterisation of the ejected particle environment is established showing that the ejection of surface particles from an active asteroid contributes to the commonly observed 'double-top' shape. Auto-navigation and Neural network based guidance is then demonstrated. The concept of achieving auto-navigation using passive line of sight measurements from poorly known objects under the influence of a common gravitational field is quantified, with results showing convergence for both the spacecraft and ejected particle position and velocity estimates. Significant improvements in performance are plausibly expected when the spacecraft begins maneuver, as observability improves quickly what is effectively temporal triangulation.

Perceptron based guidance is demonstrated by showing convergence of a trained neural network to mimic the output of the iterative Lambert's solution. Comparison of trajectories showing both solutions demonstrates the promise of this technique. Spacecraft control is explored by showing the existence of a 10 thruster placement for redundant 6 degree of freedom control, thus indicating the means to establish optimization. The development of a universal grappling scheme for non-cooperative targets and consistent with known projectile sizes is then explored and control algorithms are developed for its use. Finally, a demonstration of these technologies is executed on a air-bearing table contoured to mimic the low gravity environment of a small asteroid. The overall resulting mission design is conceptually designed and risk reduced for key technological components. 


\section{References}

[1] M.C. Nolan et al. Detection of Rotational Acceleration of Bennu Using HST Light Curve Observations. Geophysical Research Letters, 2018

[2] D.J. Scheeres et al. The dynamic geophysical environment of (101955) Bennu based on OSIRIS-REx measurements. Nature Astronomy, 2019

[3] D.J. Scheeres et al. The geophysical environment of Bennu. Icarus, 2016

[4] D.S. Lauretta et al. Episodes of particle ejection from the surface of the active asteroid (101955) Bennu. Science, 2019

[5] K. Sugiura, H. Kobayashi, and S. Inutsuka. Toward Understanding the Origin of Asteroid Geometries: Variety in Shapes Produced by Equal-Mass Impacts. Astronomy and Astrophysics, 2018

[6] D.J. Scheeres. Landslides and Mass shedding on spinning spheroidal asteroids, Icarus, 2015

[7] O.S. Barnouin et al. Shape of (101955) Bennu indicative of a rubble pile with internal stiffness. Nature Geoscience, 2019

[8] Alan W. Harris, Eugene G. Fahnestock, Petr Pravec. On the shapes and spins of "rubble pile" asteroids. Icarus 2009

[9] S. Watanabe et al. Hayabusa2 arrives at the carbonaceous asteroid (162173) Ryugu - A spinning top - shaped rubble pile. Science, 2019

[10] D.J. Scheeres et al. Dynamical Environment of Binary Near-Earth Asteroid (66391) 1999 KW4. Science 2006

[11] Guibout, V., Scheeres, D. Stability of Surface Motion on a Rotating Ellipsoid. Celestial Mechanics and Dynamical Astronomy, 2003

[12] Kevin J. Walsh. Rubble Pile Asteroids. Annual Review of Astronomy and Astrophysics 2018

[13] P. Michel, W. Benz, P. Tanga, D. C. Richardson. Collisions and gravitational reaccumulation: Forming asteroid families and satellites. Science 2001

[14] M. Hirabayashi, D. J. Scheeres. Rotationally induced failure of irregularly shaped asteroids. Icarus 2019

[15] M. Hirabayashi, D. J. Scheeres. Stress and failure analysis of rapidly rotating asteroid (29075) 1950 DA. Astrophysics 2014

[16] M. W. Busch, J. Giorgini, S. Ostro, L. Benner, R. Jurgens, R. Rose, M. Hicks, P. Pravec, P. Kusnirak, M. Ireland. Physical modeling of near-Earth Asteroid (29075) 1950 DA Icarus 2007

[17] S. J. Ostro, J.-L. Margot, L. A. M. Benner, J. D. Giorgini, D. J. Scheeres, E. G. Fahnestock, S. B. Broschart, J. Bellerose, M. C. Nolan, C. Magri, P. Pravec, P. Scheirich, R. Rose, R. F. Jurgens, E. M. De Jong, S. Suzuki. Radar imaging of binary near-Earth asteroid (66391) 1999 KW4. Science 2006

[18] M. W. Busch, S. J. Ostro, L. A. M. Benner, M. Brozovic, J. D. Giorgini, J. S. Jao, D. J. Scheeres, C. Magri, M. C. Nolan, E. S. Howell, P. A. Taylor, J.-L. Margot, W. Brisken. Radar observations and the shape of near-Earth Asteroid 2008 EV5. Icarus 2011

[19] Walsh, K. J., Richardson, D. C. \& Michel, P. Spin-up of rubble-pile asteroids: disruption, satellite formation, and equilibrium shapes. Icarus (2012).

[20] Hergenrother, C. W. et al. Operational environment and rotational acceleration of asteroid (101955) Bennu from OSIRIS-REx observations. Nature Communications (2019).

[21] Statler, T. S. Extreme sensitivity of the YORP effect to small-scale topography Icarus (2009).

[22] Minton, D. A. The topographic limits of gravitationally bound, rotating sand piles. Icarus (2008).

[23] Scheeres, D.J. et al. Dynamical configuration of binary near-earth asteroid (66391) 1999 KW4. Science (2006).

[24] Nolan, M. C. et al. Shape model and surface properties of the OSIRIS-REx target asteroid (101955) Bennu from radar and lightcurve observations. Icarus (2013).

[25] Walsh, K. J. et al. Craters, boulders and regolith of (101955) Bennu indicative of an old and dynamic surface. Nature Geoscience (2019).

[26] Sanchez, P. and Scheeres, D. J. Disruption patterns of rotating self-gravitating aggregates: a survey on angle of friction and tensile strength. Icarus 271, 453-471 (2016). 
[27] Zhang, Y. et al. Rotational failure of rubble-pile bodies: influences of shear and cohesive strengths. Astrophysics 857, 15 (2018).

[28] Delbo, M. et al. Thermal fatigue as the origin of regolith on small asteroids. Nature 508, 233-236 (2014).

[29] Keith A. Holsapple. On YORP-induced spin deformations of asteroids. Icarus 205 (2010) 430-442

[30] P Sánchez, D.J. Scheeres. Simulating asteroid rubble piles with a self-gravitating soft-sphere distinct element method model. The Astrophysical Journal, 2011

[31] D. S. Lauretta, D.N. DellaGiustina, and The OSIRIS-REx Team, The unexpected surface of asteroid (101955) Bennu Nature, March 2019

[32] Kevin J. Walsh, Derek C. Richardson \& Patrick Michel. Rotational breakup as the origin of small binary asteroids Nature, August 2008

[33] Mendel, J.M. Lessons in Digital Estimation Theory, Prentice-Hall Press, 1987, pp. 252-253.

[34] J. R. Yim, J. L. Crassidis and J. L Junkins. Autonomous Orbit Navigation Of Two Spacecraft System Using Relative Line Of Sight Vector Measurements. Advances in the Astronautical Sciences, 2005, Vol.119, pp.2447-2460

[35] S. Bhaskaran, J.E. Riedcl, S. P. Synnott, Autonomous Optical Navigation for Interplanetary Missions. SPIE Proceedings 1996.

[36] K. Hill and George H. Born. Autonomous Interplanetary Orbit Determination using Satellite to Satellite Tracking. Journal of Guidance, Control, and Dynamics Vol. 30, No. 3, May-June 2007.

[37] B.Polle, B.Frapard, X.Sembely. Autonomous Navigation Concepts for Interplanetary Missions. IFAC Proceedings Volume 37, Issue 6, June 2004, pp. 203-208.

[38] R. R. Karimi and D. Mortari. Interplanetary Autonomous Navigation Using Visible Planets. Journal of Guidance, Control, and Dynamics. Vol. 38, Issue 6, 2015, pp. 1151-1156.

[39] B. Polle, B. Frapard, J. Gil-Fernandez, E. Milic, M Graziano, J. Rebordao, P. Motrena. Autonomous Navigation for Interplanetary Missions, Performance Achievements Based on Real and Flight Images. Proceedings of the 6th interna-tional ESA Conference on Guidance, Navigation and Control Systems 17-20 October 2005

[40] J. E. Riedel, S. Bhaskaran, S. Desai, D. Han, B. Kennedy, T. McElrath, G. W. Null, M. Ryne, S. P. Synnott, T. C. Wang, R. A. Werner. Using Autonomous Navigation for Interplanetary Missions: The Validation of Deep Space I Autonav. International Conference on Low-Cost Planetary Missions May 2, 2000.

[41] S. Shemar et al. Towards practical autonomous deep-space navigation using X-Ray pulsar timing. Experimental Astronomy Vol. 42, No. 2, October 2016, pp.101-138.

[42] Li, Haiyang et al, Deep Networks as Approximators of Optimal Transfers Solutions in Multitarget Missions, Acta Astronautica Volume 166, Pages 469-481, January 2020

[43] Damien Guého. A Comparison of Parametric and Non-Parametric Machine Learning Approaches for the Uncertain Lambert Problem. AIAA Scitech 2020 Forum

[44] Yue-he Zhu, Ya-Zhong Luo. Fast Evaluation of Low-Thrust Transfers via Multilayer Perceptions. Journal of Guidance, Control and Dynamics Vol. 42, No. 12, December 2019

[45] D.S. Lauretta et al. Episodes of particle ejection from the surface of the active asteroid (101955) Bennu. Science, 2019

[46] Battin,R.H.and R.M.Vaughan. An elegant Lambert algorithm. Journal of Guidance, Control, and Dynamics 7(6), pp.662-670. 1984

[47] Engels, R. and J.Junkins, The gravity-perturbed Lambert problem: AKS variation of parameters approach Celestial mechanics 24(1), pp.3-21. 1981

[48] Marquardt, D. An Algorithm for Least-Squares Estimation of Nonlinear Parameters. SIAM Journal on Applied Mathematics Vol. 11, No. 2, pp. 431-441, June 1963.

[49] Bindel, Daniel. Six-Dimensional Thruster Actuation and Configuration Design for Spacecraft. Advances in the astronautical sciences Vol. 128, pp. 929-945, 2007. 
[50] Asher Smith and Dongeun Seo. Spacecraft Thruster Distribution Matrix for Precision 6DOF Control. AIAA Space Forum Sep 2017.

[51] Eleonora M. Botta, Inna Sharf and Arun K. Misra, Contact Dynamics Modeling and Simulation of Tether Nets for Space-Debris Capture, Journal of Guidance, Control and Dynamics January 2017.

[52] M. A. Amiri Atashgah, Hamid Gazerpour, Abolfazl Lavaei, Yaser Zarei, An active time-optimal control for space debris deorbiting via geomagnetic field. Celestial Mechanics Dynamical and Astronomy 2017.

[53] Jonghyuk Lim, Jintai Chung, Dynamic analysis of a tethered satellite system for space debris capture Nonlinear Dynamics 2018.

[54] Suril V. Shah, Inna Sharf and Arun K. Misra, Reactionless Path Planning Strategies for Capture of Tumbling Objects in Space Using a Dual-Arm Robotic System AIAA GNC Conference, August 2013

[55] Joshua J. Loughman, Overview and Analysis of the Soldier Satellite Concept for Removal of Space Debris. AIAA Space Conference, August 2010

[56] Rybus, Tomasz, and Karol Seweryn. Planar Air-bearing Microgravity Simulators: Review of Applications, Existing Solutions and Design Parameters. Acta Astronautica 120 (2016): 239-59.

[57] Tsuda, Yuichi, Makoto Yoshikawa, Takanao Saiki, Satoru Nakazawa, and Sei-Ichiro Watanabe. "Hayabusa2-Sample Return and Kinetic Impact Mission to Near-earth Asteroid Ryugu." Acta Astronautica 156 (2019): 387-93. Web.

[58] Tsuchiyama et al. "Three-Dimensional Structure of Hayabusa Samples: Origin and Evolution of Itokawa Regolith." Science

[59] Lund, Tom. "Early Exploration of the Moon Ranger to Apollo, Luna to Lunniy Korabl." 1st Ed. 2018. ed. 2018. Space Exploration. Web.

[60] Berger, Eve L, Lauretta, Dante S., Connolly, Harold C. Jr., Giacalone, Joe, Lunine, Jonathan I., and Swindle, Timothy D. Cubanite and Associated Sulfides in CI Chondrites and Comet 81P/Wild 2: Implications for Aqueous Processing (2012). Web.

[61] King, B.V, M.J Pellin, and D.S Burnett. "Investigation of Radiation Enhanced Diffusion of Magnesium in Substrates Flown on the NASA Genesis Mission." Applied Surface Science 255.4 (2008): 1455-457. Web.

[62] Everett, David F., Joshua Wood, Ronald Mink, Timothy Linn, William Willcockson, and Thomas, M. Ajluni. "OSIRIS-REx, Returning the Asteroid Sample." 2015 IEEE Aerospace Conference (2015): 2015 IEEE Aerospace Conference; 4-7 Mar. 2015; Big Sky, MT; United States. Web.

[63] Sawada et al. "Hayabusa2 Sampler: Collection of Asteroidal Surface Material." Space Science Reviews 208.1-4 (2017): 81-106. Web.

[64] Leonard Dean Vance, Erik Asphaug and Jekan Thangavelautham. "Evaluation of Mother-Daughter Architectures for Asteroid Belt Exploration," AIAA Science and Technology Forum and Exposition, 2019..

[65] Salil Rabade, Nathan Barba, Gavin Liu, Laurence Garvie and Jekan Thangavelautham. "The Case for Solar Thermal Steam Propulsion System for Interplanetary Travel: Enabling Simplified ISRU Utilizing NEOs and Small Bodies." International Astronautics Congress, 2016.

[66] Alessandra Babuscia, Thomas Choi, Johnathan Sauder, Aman Chandra, Jekan Thangavelautham, "Inflatable antenna for CubeSats: Development of the X-band prototype," IEEE Aerospace Conference 2016. 